\title{
Echo-dephased steady state free precession
}

\author{
Sunil Patil • Oliver Bieri · Klaus Scheffler
}

Received: 16 January 2009 / Revised: 17 March 2009 / Accepted: 7 April 2009 / Published online: 16 May 2009

(C) ESMRMB 2009

\begin{abstract}
Objective To introduce a novel positive contrast method for passive localization and visualization of paramagnetic susceptibility markers.

Materials and methods The novel approach is based on an echo-dephased steady-state free precession (SSFP) sequence. Gradients dephase any signal by $\pm \pi$ at the centered echotime $(\mathrm{TE}=\mathrm{TR} / 2)$ and induce a total dephasing of $\pm 2 \pi$ per pixel within TR. This ensures that background tissues do not contribute to signal formation and thus appear dark. However, within the close vicinity of the paramagnetic marker, local gradient fields compensate for the intrinsic dephasing to form an echo. Conceptual issues of gradient compensation and its visualization characteristics are analyzed. The feasibility of the proposed technique for MR-guided intravascular interventions is demonstrated using flow phantom.

Results Echo-dephased SSFP is able to localize and visualize paramagnetic marker with excellent suppression of the background signals. The flow phantom experiments concluded that reliable tracking of the interventional guidewire is feasible using echo-dephased SSFP.

Conclusion With newly introduced echo-dephased SSFP approach, accurate and reliable visualization of paramagnetic interventional device is feasible.
\end{abstract}

Keywords Positive contrast · Passive tracking · Interventional MRI $\cdot$ SSFP $\cdot$ Susceptibility

S. Patil $(\varangle) \cdot$ O. Bieri $\cdot$ K. Scheffler

Division of Radiological Physics, Department of Medical Radiology, University of Basel Hospital, Petersgraben 4,

4031 Basel, Switzerland

e-mail: sunil.patil@unibas.ch

\section{Introduction}

Magnetic field inhomogeneities, for example as generated by susceptibility differences, have a strong impact on signal properties of gradient-echo sequences [1]. It is well known that strong field inhomogeneities appear as black voids (signal loss) in a typical GRE image entailing a negative contrast with respect to background signal. On the other hand, positive contrast from local susceptibility changes was first proposed by Frahm et al. [2] using local gradient compensation. Positive contrast, also known as white marker imaging has found application in interventional MRI for passive depiction and tracking of the interventional devices [3] or to visualize magnetically labeled cells [4]. Generally a gradient in slice direction is used to dephase background signals, whereas marker-related local gradients compensate for the dephasing to produce locally a positive contrast. One of the major prerequisites for successful passive tracking is the feasibility of image acquisitions with extended slab thickness (40-50 mm) to guarantee that the marker material always lies within the plane being imaged. However, application of dephasing gradients using extended slice thickness may also enhance other unwanted background signals, e.g. from air/tissue interfaces and thereby hampering proper marker localization and visualization. Sequence developments that further reduce background sensitivity are thus desirable and only recently a promising concept for the generation of positive contrast from local susceptibilities was proposed which is based on morphing of steady-state free precession (SSFP) [5].

In this work, we further develop the idea of gradient compensation in SSFP to locally induce high signal intensities. In the close vicinity of paramagnetic materials, strong local gradient fields may compensate for any sequence-related gradients to locally induce signal transitions from an echo-dephased to an echo-rephased type of SSFP [mainly 
balanced SSFP (bSSFP)] sequence. Generically resembling an unbalanced SSFP, in echo-dephased SSFP echoes are preferably dephased by $\pm \pi$ prior to readout. This not only ensures excellent cancellation of any background signals but also generic dephasing from unbalanced SSFP greatly reduces unwanted background signal enhancements. It will be demonstrated that due to the higher in-plane resolution as compared to slice resolution, local gradient compensation in echo-dephased SSFP is likely to take place only within the close vicinity of the paramagnetic marker material. Conceptual issues of gradient compensation and its signal properties are analyzed and discussed. Finally to demonstrate the feasibility of proper marker visualization and localization using echo-dephased SSFP, reliable tracking of MR compatible interventional guidewire having paramagnetic material on it is demonstrated in a simple flow phantom.

\section{Materials and methods}

In order to illustrate the conceptual issues of echo-dephased SSFP, numerical simulations, data analysis and visualization were done using Matlab 2006a (The MathWorks, Inc.). Measurements and calibrations were performed on a Siemens 1.5 T Espree system (Siemens Healthcare, Erlangen, Germany). Unless otherwise stated, images were acquired in the coronal direction with readout and dephasing gradients applied along the main magnetic field. A matrix of $256 \times 256$ covering a FOV of $200 \mathrm{~mm}$ was used with a slice thickness of $40 \mathrm{~mm}$. The maximum available bandwidth/pixel for a given TR was utilized. No averages were taken for any of the images displayed.

Local gradient field effect

A small paramagnetic material (for example, stainless steel) induces spatially dependent local gradient perturbations $(\Delta G)$ derived from the partial derivatives of the field offsets $\left(\Delta B_{z}\right)$ according to [3]

$$
\begin{aligned}
\Delta G_{z} & =3 c z \frac{-3 x^{2}-3 y^{2}+2 z^{2}}{\left(x^{2}+y^{2}+z^{2}\right)^{7 / 2}} \\
\Delta G_{x} & =-3 c x \frac{x^{2}+y^{2}-4 z^{2}}{\left(x^{2}+y^{2}+z^{2}\right)^{7 / 2}},
\end{aligned}
$$

with $c=\frac{B_{0} \cdot \Delta \chi \cdot V}{4 \pi}, B_{0}$ is the main magnetic field, $\Delta \chi$ is the susceptibility difference between the marker material and the surrounding tissues and $\Delta \chi V\left(\mathrm{~m}^{3}\right)$ characterizes the local magnetic dose (LMD) [6]. The $\Delta G_{y}$ shows similar spatial dependence as that of $\Delta G_{x}$ due to radial symmetry of the $\Delta B_{z}$.
Depending upon the direction of the slice orientation one of these field perturbations may compensate for imaging related dephasing gradients. For example, if the slice orientation is along coronal direction, $\Delta G_{z}$ may act as a compensating gradient for any gradient applied along the main magnetic field. In the following, we will further develop the idea of compensation of echo-dephasing gradients in SSFP.

\section{Echo-dephased SSFP}

Generally within any repetition time the phase evolution $\phi_{G}$ of an isochromat at position $r(x, y, z)$ in the presence of a gradient $G$ is given by [7]

$\phi_{G}:=\gamma \int_{0}^{\mathrm{TR}} G(t) \cdot r \mathrm{~d} t$,

For SSFP, the dispersion of phase evolution $\phi_{\mathrm{G}}$ within one voxel is constant and is equal to either $2 \pi$ (SSFP-FID or SSFP-ECHO [8]) or zero (bSSFP). In echo-dephased SSFP (shown in Fig. 1a) the net phase dispersion $\phi_{G}$, is preferably set equal to $4 \pi$ /voxel. This is achieved by modifying balanced gradient pattern of the bSSFP sequence along the readout direction. The pre-phasing gradient present before the readout as well as rephasing gradient present after the readout gradient have the same polarity as that of readout gradient. Thus there is no refocusing of the readout gradient. This global behavior of the echo-dephased SSFP ensures that none of the transversal states from background tissues contribute to the echo formation.

In the presence of the local gradients, $\Delta G$, due to the marker at position $r_{0}\left(x_{0}, y_{0}, z_{0}\right)$ the amount of dephasing modifies to

$$
\begin{aligned}
\phi_{G} & \rightarrow \phi_{G}=\gamma \int_{0}^{\mathrm{TR}}\left[G(t) \cdot r+\Delta G \cdot\left(r-r_{0}\right)\right] \mathrm{d} t \\
& =\gamma \int_{0}^{\mathrm{TR}} G(t) \cdot r \cdot \mathrm{d} t+\gamma \cdot \mathrm{TR} \cdot \Delta G \cdot\left(r-r_{0}\right)
\end{aligned}
$$

As a result, different fractional compensation leads to different signal properties of echo-dephased SSFP (Fig. 1b). At certain distance (indicated as [1] in Fig. 1b), $\Delta G$ fully compensates the sequence related intrinsic dephasing $\left(\left|\phi_{G}\right|=\right.$ 0 ) to form a bSSFP type of echo similar to the morphing SSFP concept [5]. With increasing distances, gradient compensation further decreases to yield a dephasing of $\left|\phi_{G}\right|=$ $2 \pi /$ voxel (at some specific position [2] in Fig. 1b). Here, the echo is a summation of different dephasing paths within the voxel. $\Delta G$ tends to zero for $r \gg r_{0}$, leading to $\left|\phi_{G}\right|=$ $4 \pi /$ voxel as shown in Fig. 1a and indicated as position [3] in 


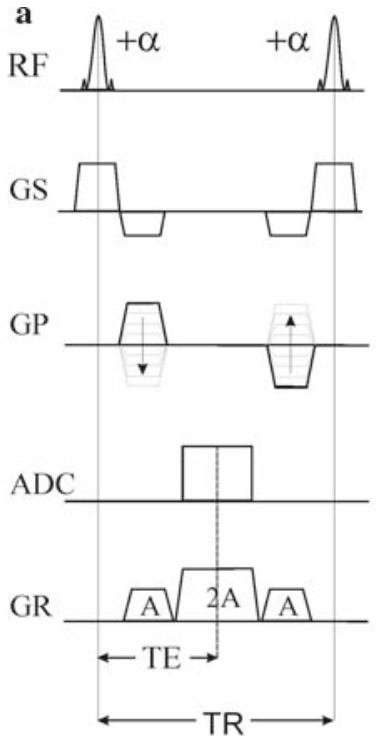

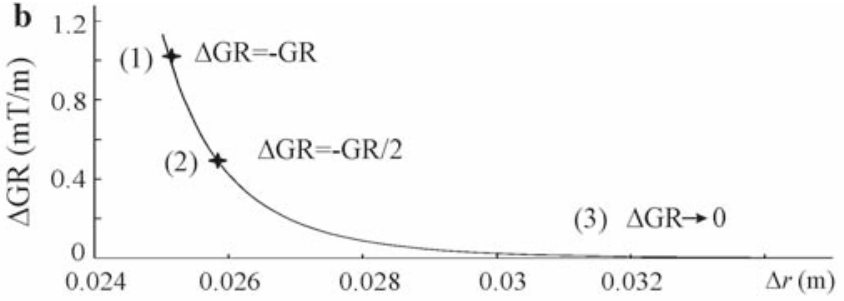
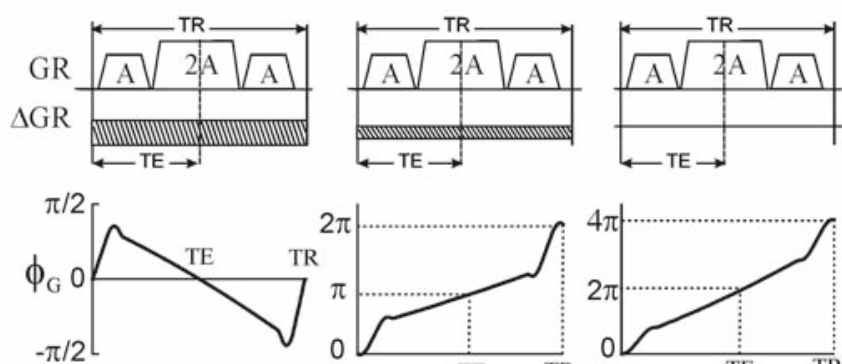

(1) $\phi_{0}=0$

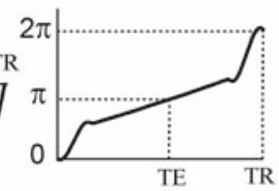

(2) $\phi_{0}=2 \pi$

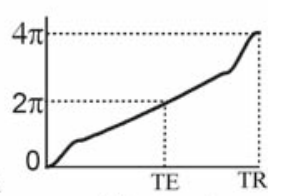

(3) $\phi \rightarrow 4 \pi$
Fig. 1 The working principle of echo-dephased SSFP. a Sequence diagram. Echo-dephased SSFP dephases spins by $4 \pi$ /voxel along the readout direction (total area: 4A). This ensures complete suppression of the background. $\mathbf{b}$ The signal behavior at $\mathrm{TE}=\mathrm{TR}$ in the presence of local gradient fields $(\Delta G)$, e.g. as induced by the paramagnetic ring marker. At certain spatial locations [1-3], $\Delta G$ may fully, partially or not com- pensate the sequence-related dephasing gradient to form different types of SSFP echo: At [1]: balanced SSFP ( $\Delta G$ may fully compensate an amount equal to G), at [2]: averaged balanced SSFP (at location further away from marker, $\Delta G$ may compensate an amount equal to $\mathrm{G} / 2$ ) and at [3]: echo-dephased SSFP (with further increase in the distance away from the marker, the sequence may exhibit global behavior shown in a)
Fig. 1b. Thus in this region no compensation of the sequencerelated dephasing takes place.

For a uniform distribution of different dephasing angles $\mathrm{p}\left(\phi_{G}\right)$ and in the presence of various off-resonances $\phi_{0}$, the steady-state signal $S(t=\mathrm{TE})$ is given by [9]:

$S(\mathrm{TE}) \propto \int p\left(\phi_{G}\right) \cdot M_{+}\left(\phi_{G}+\phi_{0}\right) \cdot e^{-\mathrm{TE} / T_{2}}$

$$
\times e^{i \cdot\left(\phi+\phi_{0}\right) \cdot \mathrm{TE} / \mathrm{TR}} \mathrm{d} \phi .
$$

Here $M_{+}(\phi)$ is the transverse magnetization after the excitation pulse and is given by,

$$
\begin{aligned}
& M_{+}(\phi):=M_{y}+i M_{x}=M_{0} \cdot \sin (\alpha) \\
& \times \frac{\left(1-E_{1}\right)\left(1-E_{2} e^{-i \phi}\right)}{C \cdot \cos (\phi)+D}, \quad \text { where } E_{1,2}:=e^{-\mathrm{TR} / T_{1,2}}
\end{aligned}
$$

and

$C:=E_{2}\left(E_{1}-1\right)(1+\cos (\alpha))$,

$D:=\left(1-E_{1} \cos (\alpha)\right)-\left(E_{1}-\cos (\alpha)\right) E_{2}^{2}$.

In order to guide the development of echo-dephased SSFP, simulations of $S(\mathrm{TE})$ using Eq. 4 were performed at different TE values resembling the well-known SSFP sequences namely SSFP-FID $(\mathrm{TE}=0)$, bSSFP $(\mathrm{TE}=\mathrm{TR} / 2)$ and SSFPECHO $(\mathrm{TE}=\mathrm{TR})$ as a function of fractional gradient compensation $\left(\phi_{G}\right)$ varying in the range of $[-4 \pi: 4 \pi]$ and off-resonance $\left(\phi_{0}\right)$ varying from -540 to $540 \mathrm{~Hz}$. The other imaging parameters used for simulations were: $\mathrm{TR}=3 \mathrm{~ms}$,
$T_{1}=300 \mathrm{~ms}$ and $T_{2}=100 \mathrm{~ms}$. To further demonstrate the signal characteristics of the echo-dephased SSFP dependencies of the steady state signal on different TRs and relaxation times were evaluated. This was achieved by plotting the difference between the steady-state signal, $S$ (TE) obtained at two different TRs (TR $=3$ and $20 \mathrm{~ms}$ ) and at two different sets of relaxation times $\left(T_{1}=1,200 \mathrm{~ms}, T_{2}=400 \mathrm{~ms}\right.$ and $\left.T_{1}=300 \mathrm{~ms}, T_{2}=100 \mathrm{~ms}\right)$. In all simulations, the near optimal flip angles $\left(\alpha_{\text {opt }} \approx \cos ^{-1}[(\varepsilon-1) /(\varepsilon+1)], \varepsilon=T_{1} / T_{2}\right.$; see $[10,11])$ were used.

To visualize the effect of local gradient fields (see Eq. 1) surrounding the paramagnetic marker during typical twodimensional (2D) magnitude image acquisition, numerical simulations were carried out for a coronal slice. The paramagnetic marker of radius $1 \mathrm{~mm}$ was assumed to be placed at the center. The signal intensity calculations for bSSFP $\left(\left|\phi_{\mathrm{G}}\right|=\right.$ 0/voxel) and echo-dephased SSFP $\left(\left|\phi_{\mathrm{G}}\right|=4 \pi /\right.$ voxel $)$ were carried out over the FOV of $40 \mathrm{~mm}$ and slice thickness $40 \mathrm{~mm}$ using Eq. 4 in the presence of $\Delta G$. The readout was placed along the main magnetic field. The other imaging parameters were: $\mathrm{TE} / \mathrm{TR}=2.5 / 5.0 \mathrm{~ms}$ and $T_{1} / T_{2}=300 / 200 \mathrm{~ms}$.

Guidewire and phantom

An MR-compatible guidewire made up of Polyetherethertone synthetic polymer core with a diameter of $0.7 \mathrm{~mm}$ and compatible with $0.035^{\prime \prime}$ catheter was used [12]. The paramagnetic material made up of stainless steel alloy was wrapped 
around the guidewire near its tip with approximate dimensions $1 \mathrm{~mm}$ in height and diameter. Frequency measurements yield a LMD of $\Delta \chi V=1 \times 10^{-11} \mathrm{~m}^{3}$ (in SI units). The guidewire was inserted into a custom-built phantom with $11 \mathrm{~mm}$ diameter tubes to model the blood flow, positioning and tracking of the guidewire in large vessels. The tubes were immersed in a gadolinium (Gd)-doped water and were surrounded by a $2 \%$ agarose gel (v/v) doped with $0.5 \mathrm{mM}$ copper sulphate $\left(\mathrm{CuSO}_{4}\right)$ concentration to closely resemble the relaxation times of muscle tissues for which $T_{1}=900 \mathrm{~ms}$ and $T_{2}=50 \mathrm{~ms}$. A 7F introducer sheath was attached to the tubes to facilitate the introduction and manipulation of the guidewire within the tubes.

\section{Passive tracking experiments}

The passive tracking experiments followed a standard procedure: First, a roadmap of the flow phantom was obtained resembling the vasculature structure of human leg (see Fig. 6). Second, positive contrast images from echo-dephased SSFP are merged with the roadmap in near real time in the following manner: In a first step, both roadmap and echo-dephased SSFP images were converted into binary masks by thresholding. In a second step, the two masks were multiplied to confine the marker position within the roadmap, which was finally merged with the original roadmap image. The tubes of the phantom were connected to a pump (14-166; Cobe Laboratories Inc., Lakewood, CO, USA) and the flow was set to $4 \mathrm{~mL} / \mathrm{s}$. The circulating water was doped with $0.5 \mathrm{~mL}$ Gd-DOTA (Dotarem; Guerbet, Roissy CDG Cedex, France) having a $T_{1}$ relaxation time similar to blood $(1,270 \mathrm{~ms})$.

The 2D roadmap was generated based on an inversionrecovery SSFP-FID sequence, as described in [12] using $\mathrm{FOV}=30 \mathrm{~cm}, \mathrm{TR} / \mathrm{TE}=4.21 / 2.11 \mathrm{~ms}, \alpha=40^{\circ}$ and bandwidth/pixel $=720 \mathrm{~Hz}$. Echo-dephased SSFP images were acquired with essentially the same FOV having TR/TE = $3.5 / 1.75 \mathrm{~ms}$, bandwidth/pixel $=590 \mathrm{~Hz}$ and partial Fourier factor of 5/8. Parallel imaging technique, GRAPPA with an acceleration factor of 3 and 12 reference lines were also utilized, yielding a total image acquisition time for positional marker update of around $250 \mathrm{~ms}$.

\section{Results}

This section begins with the description and elaboration of the results of the numerical simulations of $S(\mathrm{TE})$ using Eq. 4 and 2D imaging of the bSSFP and echo-dephased SSFP. Next we describe the phantom experiments to demonstrate the proof of principles of echo-dephased SSFP. This is followed by detailed description and analysis of the spatial accuracy and localization characteristics of the echo-dephased SSFP.
Finally the results of the passive tracking experiments in a flow phantom conclude the section.

\section{Signal characteristics of echo-dephased SSFP}

In Fig. 2a echo amplitudes at different TEs (TE $=0, T R / 2$ and TR) as a function of fractional compensation $\left(\phi_{\mathrm{G}}\right)$ and offresonance frequency $\left(\phi_{0}\right)$ are displayed. It can be seen that in the region of around $-2 \pi \leq \phi_{\mathrm{G}} \leq 2 \pi$, that is when local gradient fields compensate the sequence related dephasing gradients (partially or fully; see cases [1] and [2] in Fig. 1b), the signal amplitude is conserved $\left(M_{x y}>0.25\right)$ at offresonant frequencies $\left(\phi_{0} \sim \pm 250 \mathrm{~Hz}, \pm 500 \mathrm{~Hz}\right)$. As the fractional gradient compensation $\phi_{G}$ makes a transition from $\phi_{G} \approx 2 \pi$ towards $\phi_{G} \rightarrow 4 \pi$ (no compensation; see case [3] in Fig. 1b), the signal intensity drops considerably $\left(M_{x y}<\right.$ 0.15 ). As a result, the difference between the signal intensities of these two regions and thereby contrast, increases dramatically for off-resonant frequencies when dephasing of $4 \pi /$ voxel is set in a SSFP sequence. The comparison of the contrast to noise ratio (CNR) of the signal, $S(\mathrm{TE})$ reveals that the CNR of the signal $S(\mathrm{TE}=\mathrm{TR} / 2)(\mathrm{CNR}>100)$ is dramatically higher than the signal, $S(\mathrm{TE}=0)$ and $S(\mathrm{TE}=\mathrm{TR})$ $(\mathrm{CNR} \approx 2)$. This behavior may be attributed to complete refocusing of the magnetization at $\mathrm{TE}=\mathrm{TR} / 2$ for phase in the range of $\pm 0.8 \pi(9)$ and immunity of the signal to field inhomogeneities especially at short TRs. Thus the bSSFP type of sequence with net gradient area $\left|\phi_{G}\right|$ equal to $4 \pi /$ voxel is best suited for echo-dephased SSFP. In Fig. $2 b$ and $c$, the intensity variation, $\Delta M_{x y}$ as a function of the difference between the steady-state signal obtained at two different TRs (TR $=3$ and $20 \mathrm{~ms})$ and for two different sets of relaxation times $\left(T_{1} / T_{2}=\right.$ $1,200 \mathrm{~ms} / 400 \mathrm{~ms}$, and $T_{1} / T_{2}=300 \mathrm{~ms} / 100 \mathrm{~ms}$ ), respectively is shown. The $\Delta M_{x y}$ obtained at $\mathrm{TE}=0$, decreases with an increase in TR and decrease in relaxation times, whereas at $\mathrm{TE}=\mathrm{TR}$ it increases with an increase in TR and decrease in relaxation times. This is in contrast to the $\Delta M_{x y}$ obtained at $\mathrm{TE}=\mathrm{TR} / 2$ which remains almost constant with a variation in TR as well as the ratio of relaxation times $\left(T_{1} / T_{2}\right)$. These properties reveal that in echo-dephased SSFP sequence, if the signal is obtained at TE $=\mathrm{TR} / 2$, the positive contrast generation due to paramagnetic material is independent of TR as well as the ratio of the relaxation times $\left(T_{1} / T_{2}\right)$ of the sample being imaged.

Figure 3 a depicts the simulated bSSFP magnitude image exhibiting the signal intensity pattern of typical susceptibility artifacts (black voids) due to paramagnetic marker. As noted earlier, the phase dispersion $\phi_{G}$, within a voxel for bSSFP sequence is zero. However, the local gradient field induced by paramagnetic marker introduces additional dephasing within its surrounding tissues. As a result, the signal intensity drops as a function of additional dephasing. In Fig. 3b, simulated echo-dephased SSFP magnitude 

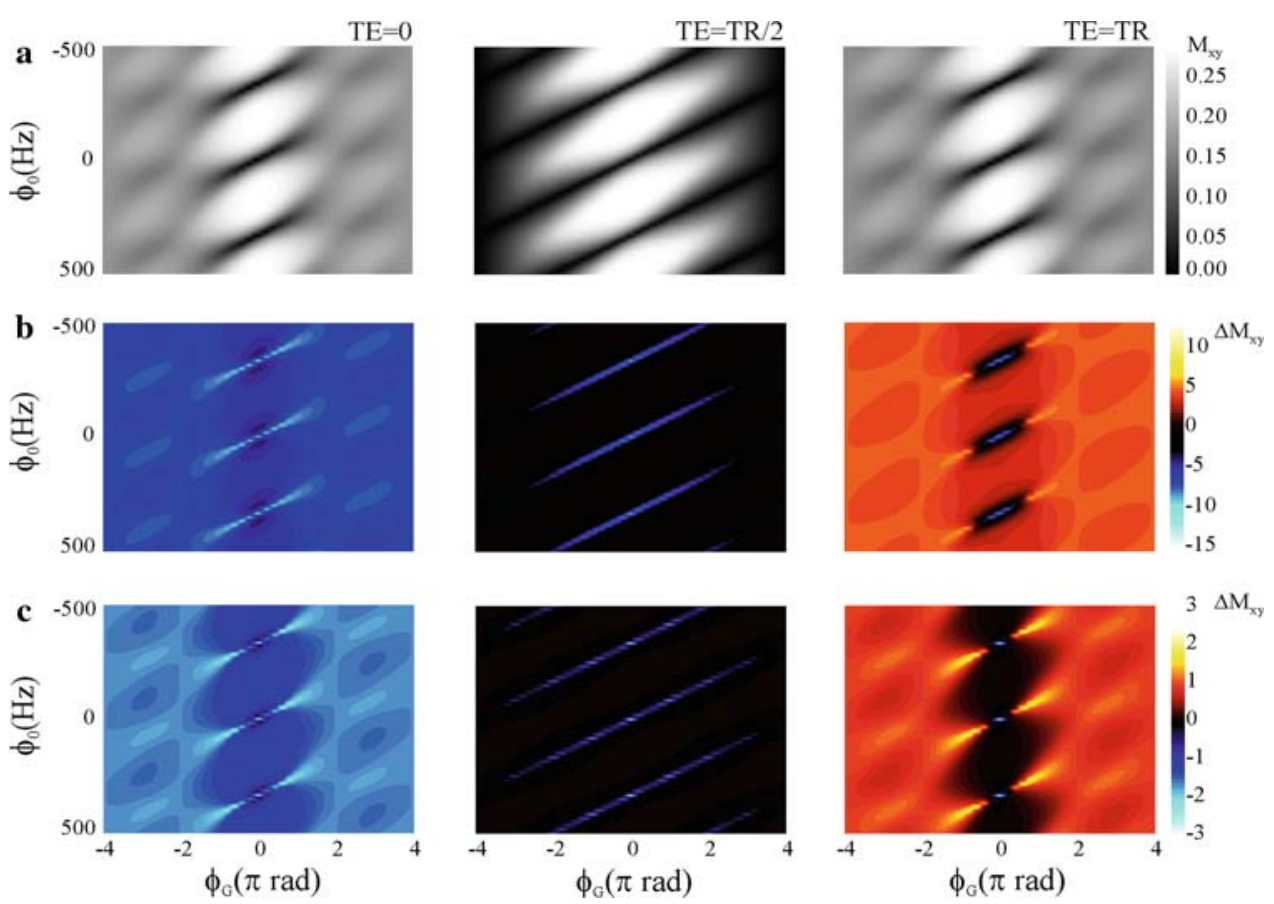

Fig. 2 a Signal amplitudes $S(\mathrm{TE})$ at various TEs $(\mathrm{TE}=0, \mathrm{TR} / 2$, TR) resembling the well-known SSFP sequences namely SSFP-FID, bSSFP and SSFP-ECHO, respectively, as a function of $\phi_{\mathrm{G}}$ varying in the range of $[-4 \pi: 4 \pi]$ and off-resonance frequencies $\left(\phi_{0}\right)$ varying from -540 to $540 \mathrm{~Hz}$ at optimal flip angle. The other imaging parameters were: TR $=3 \mathrm{~ms}, T_{1}=300 \mathrm{~ms}$ and $T_{2}=100 \mathrm{~ms}$. In the region of around $-2 \pi \leq \phi_{\mathrm{G}} \leq 2 \pi$, that is when local gradient fields compensate the sequence related dephasing gradients (partially or fully), the signal amplitude is conserved $\left(M_{\mathrm{xy}}>0.25\right)$ at off-resonant fre-
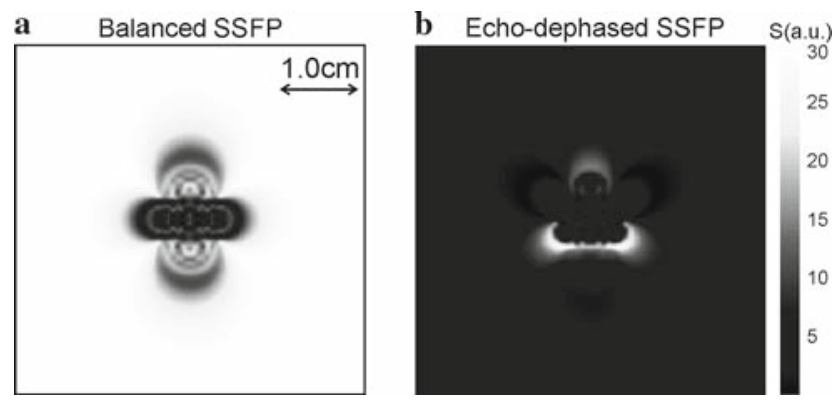

Fig. 3 Numerical simulation of the a balanced SSFP and b echodephased SSFP sequence for a coronal slice with readout along the main magnetic field over the FOV of $40 \mathrm{~mm}$ at optimal flip angle in the presence of the paramagnetic marker located at the center. The other imaging parameters were: $\mathrm{TE} / \mathrm{TR}=2.5 \mathrm{~ms} / 5 \mathrm{~ms}, T_{1} / T_{2}=300 \mathrm{~ms} / 200 \mathrm{~ms}$, slice thickness $40 \mathrm{~mm}$. The signal level $(S)$ is shown in arbitrary units (a.u.)

image is shown. In echo-dephased SSFP, the local gradient field competes with deliberately introduced sequence related dephasing gradients at specific spatial locations leading to positive signal (hyper intense blobs in Fig. 3b) with respect to its background. The distance between the two prominent hyper intense blobs was found to be $\sim 1 \mathrm{~cm}$ for the simulation parameters used. quencies $\left(\phi_{0} \approx \pm 250 \mathrm{~Hz}, \pm 500 \mathrm{~Hz}\right)$. As the fractional gradient compensation $\phi_{\mathrm{G}}$ makes a transition from $\phi_{\mathrm{G}}=2 \pi$ towards $\phi_{G} \rightarrow 4 \pi$ (no compensation), the signal intensity $(S(\mathrm{TE}))$ drops considerably $\left(M_{\mathrm{xy}}<0.15\right)$. b The signal difference between $S(\mathrm{TE})$ at two different TRs (TR $=3$ and $20 \mathrm{~ms}$ ) and $\mathrm{c}$ ) at two different sets of relaxation times $\left(T_{1} / T_{2}=1,200 \mathrm{~ms} / 400 \mathrm{~ms}\right.$ and $\left.T_{1} / T_{2}=300 \mathrm{~ms} / 100 \mathrm{~ms}\right)$. Thus, if the signal is obtained at $\mathrm{TE}=\mathrm{TR} / 2$, the positive contrast generation due to paramagnetic material is independent of TR as well as the ratio of relaxation times $\left(T_{1} / T_{2}\right)$ of the sample being imaged

\section{Phantom experiments}

The overview of the experimental flow phantom is illustrated in Fig. 4a. The bSSFP image showing the flow tubes and local field perturbations (see arrow in Fig. 4a) from the inserted paramagnetic marker material localizing the tip of the guidewire is displayed. Figure $4 \mathrm{~b}$ and $\mathrm{c}$ depicts the sample images obtained using echo-dephased SSFP with similar sequence parameters as that of bSSFP (Fig. 4a) but with different slice thicknesses. For comparison, the image obtained using white marker imaging method at a slice thickness of $40 \mathrm{~mm}$ (Fig. 4d) is also shown.

\section{Localization characteristics of echo-dephased SSFP}

To characterize the localization accuracy of echo-dephased SSFP, the distance d between the centers of the two prominent hyper-intense blobs was analyzed as a function of TR within about 4.7 to $100 \mathrm{~ms}$.

From Eq.3, phase evolution from local gradients as induced by paramagnetic marker materials are directly proportional to TR. Thus, with increasing TR, gradient 


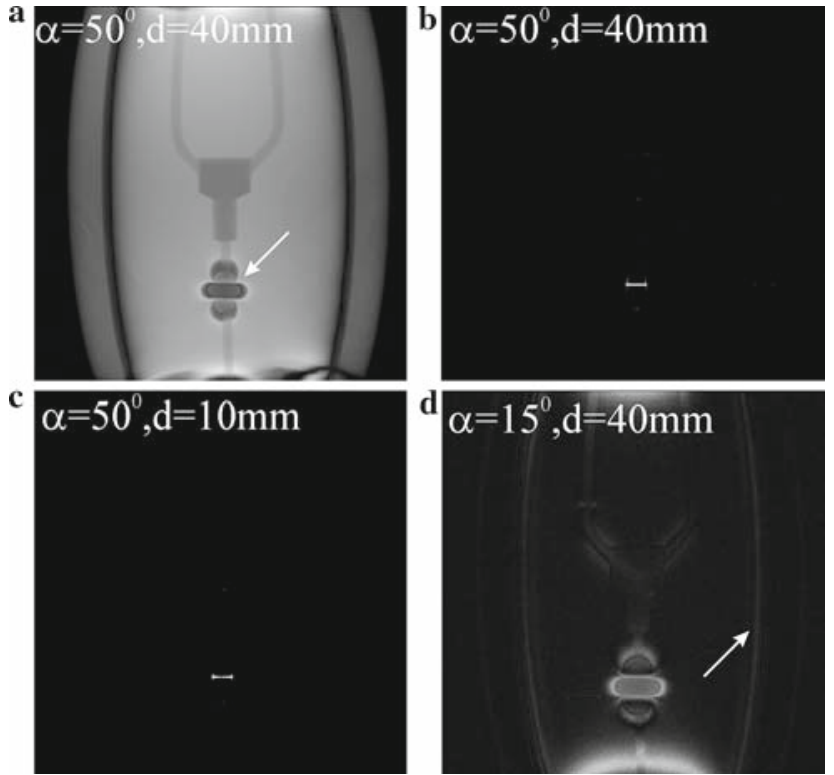

Fig. 4 In-vitro phantom images. a An experimental phantom image in the coronal direction using bSSFP sequence showing the flow tubes and black void (see arrow) resembling dipolar form due to local field perturbations induced by paramagnetic marker material wrapped around inserted guidewire is clearly visible (The imaging parameters were $\alpha=$ $50^{\circ}, \mathrm{TR}=4.7 \mathrm{~ms}$, slice thickness $=40 \mathrm{~mm}$, matrix $\left.=256 \times 256\right)$. b An echo-dephased SSFP image obtained with the same parameters and c with a slice thickness $=10 \mathrm{~mm}$. The net dephasing of $4 \pi /$ voxel ensures that background is completely dephased and appears in intense black whereas within the close vicinity of the marker a hyper intense signal is formed from local gradient compensation (consistent with the theoretical predictions). d Illustrative sample image using a white-marker imaging method with $0 \%$ slice rephasing at slice thickness $=40 \mathrm{~mm}$. The other imaging parameters were: $\alpha=15^{\circ}, \mathrm{TR}=13 \mathrm{~ms}, \mathrm{TE}=10 \mathrm{~ms}$

compensation is located further and further away from the marker's position according to [5]

$r(\mathrm{TR})=\sqrt[4]{\mathrm{TR}}$

In Fig. 5, localization accuracy of echo-dephased SSFP is analyzed. From Eq. 7, the distance $(r)$ from the perturbation source, where local gradient compensation takes place, is expected to scale proportional to TR in a double logarithmic plot with a slope of $1 / 4$. This is in good correspondence with the experimental results from hyper intensity distance analysis displayed in Fig. 5, yielding a slope of 0.287 close to expected value.

\section{Passive tracking}

Selective frames of passive guidewire tracking from echodephased SSFP are shown in Fig. 6 at various stages of the guidewire marker positions. Here, Fig. 6a shows the images obtained by applying the echo-dephased SSFP only. It may be interesting to note that because of the ring-like structure of the marker material its visualization is independent of its

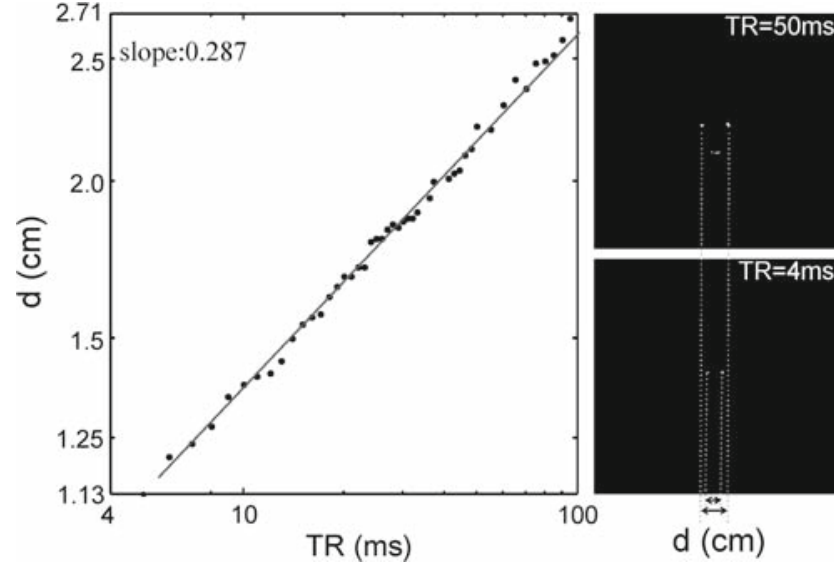

Fig. 5 Accuracy of the marker localization using echo-dephased SSFP: Variation in distance $(d)$ between two hyper-intense blobs with respect to TR is plotted using double logarithmic scale. The slope of the fitted line was 0.287 . A minimum distance of around $1.2 \mathrm{~cm}$ was observed with shorter TRs (4-5 ms) indicating its proximity to the marker material. Calculation of the distance is shown on the adjacent image insets
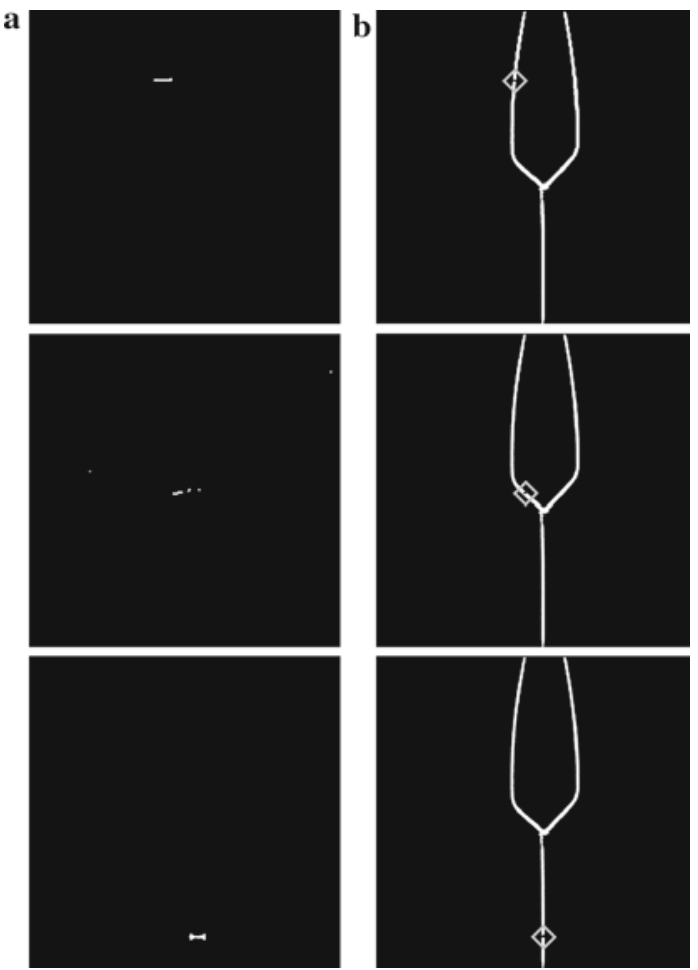

Fig. 6 Passive tracking of the interventional guidewire using echodephased SSFP technique. a The positive contrast marker images obtained using echo-dephased SSFP technique at different stages of the guidewire tracking in a flow phantom. $\mathbf{b}$ The overlay of the marker position (surrounded by gray diamonds) onto previously acquired 2D roadmap. The roadmap was acquired using IR-FISP sequence

orientation with respect to main magnetic field $\left(B_{0}\right)$. This is clearly evident from the middle panel of the Fig. 6a in which positive contrast generated by the marker material is 
clearly visible even though it is aligned at an acute angle with respect to main magnetic field. The corresponding fusion of the previously acquired roadmap and the echo-dephased SSFP image are shown in Fig. 6b. Here, the position of the marker was updated at about four frames per second. The temporal resolution could be increased up to eight frames per second by maintaining the FOV only over vessel region and reducing the in-plane resolution without significant degradation of the marker depiction.

\section{Discussion}

It was demonstrated that the dephasing by $4 \pi /$ voxel in the readout direction (from bottom to top in the images displayed; see Fig.4) ensures a completely dephased background and hyper intense signals appear only in the close vicinity of the paramagnetic marker material due to local field compensation; being consistent with theoretical predictions (see Figs. $1 \mathrm{~b}$ and $3 \mathrm{~b}$ ). Using white marker imaging method at lower slice resolutions (slice thickness $=40 \mathrm{~mm}$ ) partial volume effects due to tissue interfaces (see arrow in Fig. 4d), inherent main magnetic field inhomogeneities at the two extremes of the magnet appears with prominent positive contrast. This may hamper the accurate discrimination of the paramagnetic marker material from other unwanted background signal enhancements. Recently, it has been shown that by applying equal and opposite dephasing gradients and performing modulus subtraction of the resultant images partial volume effects can be eliminated [13]. However, this doubles the acquisition time and in addition reduces the SNR of the resultant image.

Appropriate matching of the marker position and its localization in the image is one of the major prerequisite of MR-guided interventions. In the echo-dephased SSFP sequence, compensation of the sequence-related dephasing gradients by the local gradient fields induced due to paramagnetic marker always takes place outside of the marker. and the spatial accuracy of the positive contrast generated by echo-dephased SSFP has to be determined. Reconsidering Eq. 3, the phase evolution of spins $\left.\left(\phi_{G}(x)\right)\right)$ is expressed as (e.g., in the $x$-direction):

$$
\begin{gathered}
\phi_{G}(x)=\gamma \cdot \operatorname{TR} \cdot\left(G_{x} \cdot x+\Delta G_{x} \cdot\left(x-x_{0}\right)\right)=\gamma \cdot G_{x} \\
\quad \times \mathrm{TR} \cdot\left(x \cdot\left(1+\frac{\Delta G_{x}}{G_{x}}\right)-x_{0} \frac{\Delta G_{x}}{G_{x}}\right)
\end{gathered}
$$

Noticing that $k_{x}=\gamma \cdot G_{x} \cdot$ TR, the position $x$ will be encoded at position $x^{\prime}$ in the reconstructed image [10] according to

$$
x^{\prime}=x \cdot\left(1+\frac{\Delta G_{x}}{G_{x}}\right)-x_{0}\left(\frac{\Delta G_{x}}{G_{x}}\right)
$$

Typically $G_{x} \sim 10 \mathrm{mT} / \mathrm{m}$ yielding $\gamma \cdot G_{x} \cdot x=420 \mathrm{~Hz} / \mathrm{mm}$, whereas frequency offsets from paramagnetic markers are in the range of $500 \mathrm{~Hz}$ at $1 \mathrm{~cm}$ distance for a given LMD at $1.5 \mathrm{~T}$. As a result when $G_{x}$ fully compensate for $\Delta G$ at the iso-center, it leads to positional error of the marker in the range of 1 pixel. However, as one goes further away from the iso-center, spatial encoding falls in higher audio frequency range (i.e., when $G_{x} \gg \Delta G_{x}$, see Eq.9) thereby making $x \approx x^{\prime}$. Thus in echo-dephased SSFP for a complete gradient compensation scheme (for example at position [1] in Fig. 1b), signal contribution comes from spatially encoded off-resonance frequencies.

The hyper-intense blobs in images produced by echodephased SSFP are within the close vicinity of the marker; however, it should be recognized that these blobs always lie in the surrounding of the actual marker position. The distance between the exact location of the marker and any one of the hyper-intense blobs is same and the mid-point of the distance between two hyper-intense blobs should lie on the interventional device. Hence, the analysis of distance between two hyper-intense blobs as function of TR was performed (see Fig. 5). It can be inferred from this analysis is that in echodephased SSFP the distance $d$ can be minimized for a given LMD by keeping TR as short as possible. A minimum distance of around $1.2 \mathrm{~cm}$ was observed at shorter TRs (4-5 ms) being consistent with theoretical predictions (see Fig. 3) and indicating its good proximity $(\sim 0.6 \mathrm{~cm})$ to the marker material. The fact that the proximity of the localization produced by echo-dephased SSFP to the physical position of the marker is directly proportional to TR for a given LMD makes this technique suitable for MR-guided interventions. This may lead to further increase in temporal resolution, which is currently one of the major obstacles of the utilization of passive tracking in practice. Also the positive signal contrast of the echo-dephased SSFP may benefit from the fact that the bSSFP sequences are less immune to main magnetic field inhomogeneities at low TRs.

In MR-guided interventions, passive tracking techniques utilizes either small paramagnetic materials [14] or contrast agents [15] for visualization of interventional devices but reliable localization and visualization of marker materials with respect to background is a major challenge. In this work, it was successfully demonstrated that the tracking of paramagnetic marker materials based on echo-dephased SSFP is feasible and achieves accurate and reliable marker localization with excellent background suppression.

Thanks to excellent background suppression due to echodephased SSFP method, and in contrast to other passive tracking techniques, there was no need to perform baseline subtraction [16] or additional post-processing [12]. This allowed for fast calculation of marker positions and thus led to only insignificant delays between the actual image acquisition and the corresponding update of the roadmap. Generally, 
any baseline subtraction method may suffer from background signal enhancement in presence of motion, whereas additional post-processing such as gradient signal maxima calculation as in [12] may be unsuitable for real time display of the images. Thus, present approach with its high contrast characteristics and better proximity to the marker material may be suitable for real time passive interventional MRI.

The previously proposed methods; morphing SSFP [5] and susceptibility gradient mapping (SGM) [17] also exploits the local gradient perturbations induced by the marker material in their immediate surrounding. In morphing SSFP, gradient perturbations compete with sequence-related dephasing gradients to induce a transition from SSFP-echo to balanced SSFP. In addition, background suppression, and thus positive contrast, is achieved by using the intrinsic high signal difference between bSSFP and SSFP-ECHO, especially for low flip angles in combination with long TR. In contrast in echo-dephased SSFP, echo-dephasing ensures excellent background suppression even at large flip angles with short TR. Since echo-formation is an amalgamation of SSFP-FID, SSFP-ECHO and bSSFP (see Fig. 1b), it shows similar sensitivity as these three SSFP sequences to flip angle variation as well as different types of off-resonances. The major advantage of echo-dephased SSFP is its short TR, providing a positive contrast region in the very close vicinity of the marker material with high temporal resolution.

The SGM method employs a localized short term Fourier transform method onto a gradient echo dataset for mapping local marker gradients along all three spatial directions ( $x, y$ and $z$ ) yielding a positive contrast image. SGM is thus based on the echo-shift in k-space, whereas echo-dephased SSFP selectively acquires shifted echoes along the readout similar to 1D SGM. However, from interventional application point-of-view, some of the current limitations of SGM method are: the positive contrast generation partially relies on TE value [18], in-phase TE is needed to avoid water-fat boundary artifacts and being post-processing method makes it computationally cumbersome.

Similar to all passive positive contrast methods, other sources of large-scale susceptibility gradients such as lungliver interfaces may appear with positive contrast in echodephased SSFP as well. A special care must therefore be taken to avoid such "false positives". For example, in current application marker position search space was confined within the vicinity of the vascular structure. It should also be noted that, since in this method marker position is updated on the previously acquired roadmap some of the disadvantages of the passive tracking remains when motion enters the game. However, the area of application for which these methods are being developed is the interventional peripheral MR angiography (ipMRA), where there is a low risk of or limited patient motion. Hence, the presented method may find prominent application to perform these interventions.

\section{Conclusion}

A novel concept for the generation of positive contrast from local susceptibilities, termed echo-dephased SSFP was proposed. In principle, echo-dephased SSFP is an unbalanced SSFP type of sequence, in which echoes are dephased prior to readout. We have demonstrated that this echo-dephasing approach ensures excellent cancellation of any background signals. From local gradient compensation hyper-intense signals are formed and thus a positive contrast emanates within the close vicinity of paramagnetic marker materials. Hyperintense signals are mainly of bSSFP type, which ensures sufficient signal intensity with respect to background. A reliable localization and robust marker detection was demonstrated using the proposed method. Finally, the passive guidewire tracking experiments were performed in flow phantoms suggesting suitable applicability of the echo-dephased SSFP for MR-guided interventions such as ipMRA.

Acknowledgments This work was supported by a grant from the Schweizerischen Nationalfonds (SNF 325200-118377) and Siemens Healthcare, Erlangen, Germany.

\section{References}

1. Reichenbach JR, Venkatesan R, Yablonskiy DA, Thompson MR, Lai S, Haacke EM (1997) Theory and application of static field inhomogeneity effects in gradient-echo imaging. J Magn Reson Imaging 7(2):266-279

2. Frahm J, Merboldt KD, Hanicke W (1988) Direct FLASH MR imaging of magnetic field inhomogeneities by gradient compensation. Magn Reson Med 6(4):474-480

3. Seppenwoolde JH, Viergever MA, Bakker CJ (2003) Passive tracking exploiting local signal conservation: the white marker phenomenon. Magn Reson Med 50(4):784-790

4. Mani V, Briley-Saebo KC, Itskovich VV, Samber DD, Fayad ZA (2006) Gradient echo acquisition for superparamagnetic particles with positive contrast (GRASP): sequence characterization in membrane and glass superparamagnetic iron oxide phantoms at 1.5T and 3T. Magn Reson Med 55(1):126-135

5. Bieri O, Patil S, Quick HH, Scheffler K (2007) Morphing steadystate free precession. Magn Reson Med 58(6):1242-1248

6. Yablonskiy DA, Haacke EM (1994) Theory of NMR signal behavior in magnetically inhomogeneous tissues: the static dephasing regime. Magn Reson Med 32(6):749-763

7. Scheffler K (1999) A pictorial description of steady-states in rapid magnetic resonance imaging. Concepts Magn Reson 11(5):291304

8. Gyngell ML (1988) The application of steady-state free precession in rapid 2DFT NMR imaging: FAST and CE-FAST sequences. Magn Reson Imaging 6(4):415-419

9. Scheffler K, Hennig J (2003) Is TrueFISP a gradient-echo or a spinecho sequence? Magn Reson Med 49(2):395-397

10. Haacke EMBR, Thompson MR, Venkatesan R (1999) Magnetic resonance imaging: physical principles and sequence design. Wiley, New York (2):571

11. Haacke EMBR, Thompson MR, Venkatesan R (1999) Magnetic resonance imaging: physical principles and sequence design. Wiley, New York 480 
12. Mekle R, Hofmann E, Scheffler K, Bilecen D (2006) A polymerbased MR-compatible guidewire: a study to explore new prospects for interventional peripheral magnetic resonance angiography (ipMRA). J Magn Reson Imaging 23(2):145-155

13. Seppenwoolde JH, Vincken KL, Bakker CJ (2007) White-marker imaging-Separating magnetic susceptibility effects from partial volume effects. Magn Reson Med 58(3):605-609

14. Rubin DL, Ratner AV, Young SW (1990) Magnetic susceptibility effects and their application in the development of new ferromagnetic catheters for magnetic resonance imaging. Invest Radiol 25(12):1325-1332

15. Unal O, Korosec FR, Frayne R, Strother CM, Mistretta CA (1998) A rapid 2D time-resolved variable-rate k-space sampling MR technique for passive catheter tracking during endovascular procedures. Magn Reson Med 40(3):356-362
16. Seppenwoolde JH, Bartels LW, van der Weide R, Nijsen JF, van het Schip AD, Bakker CJ (2006) Fully MR-guided hepatic artery catheterization for selective drug delivery: a feasibility study in pigs. J Magn Reson Imaging 23(2):123-129

17. Dahnke H, Liu W, Herzka D, Frank JA, Schaeffter T (2008) Susceptibility gradient mapping (SGM): a new postprocessing method for positive contrast generation applied to superparamagnetic iron oxide particle (SPIO)-labeled cells. Magn Reson Med 60(3):595603

18. Vonken EJ, Schar M, Stuber M (2008) Positive contrast visualization of nitinol devices using susceptibility gradient mapping. Magn Reson Med 60(3):588-594 